\title{
Aurora kinase A induces migration and invasion by inducing epithelial-to-mesenchymal transition in colon cancer cells
}

\author{
On-Yu Hong ${ }^{1, \#}$, Sang Yull Kang ${ }^{2, \#}$, Eun-Mi Noh ${ }^{1}$, Hong-Nu Yu ${ }^{1}$, Hye-Yeon Jang ${ }^{1}$, Seong-Hun Kim ${ }^{3}$, Jingyu Hong ${ }^{4}$, \\ Eun Yong Chung ${ }^{4, *}$ E Jong-Suk Kim ${ }^{1, *}$ \\ ${ }^{1}$ Department of Biochemistry, Institute for Medical Sciences, Jeonbuk National University Medical School, Jeonju 54907, ${ }^{2}$ Department of \\ Surgery, Research Institute of Clinical Medicine of Jeonbuk National University and Biomedical Research Institute of Jeonbuk National \\ University Hospital, Jeonju 54907, ${ }^{3}$ Division of Gastroenterology, Department of Internal Medicine, Research Institute of Clinical \\ Medicine, Jeonbuk National University Medical School and Hospital, Jeonju 54907, ${ }^{4}$ Department of Anesthesiology and Pain Medicine, \\ Bucheon St. Mary's Hospital, College of Medicine, The Catholic University of Korea, Seoul 06591, Korea
}

\begin{abstract}
Aurora kinase is a family of serine/threonine kinases intimately associated with mitotic progression and the development of human cancers. Studies have shown that aurora kinases are important for the protein kinase C (PKC)-induced invasion of colon cancer cells. Recent studies have shown that aurora kinase A promotes distant metastasis by inducing epithelial-to-mesenchymal transition (EMT) in colon cancer cells. However, the role of aurora kinase $\mathrm{A}$ in colon cancer metastasis remains unclear. In this study, we investigated the effects of aurora kinase $A$ on PKC-induced cell invasion, migration, and EMT in human SW480 colon cancer cells. Treatment with 12-O-tetradecanoylphorbol13-acetate (TPA) changed the expression levels of EMT markers, increasing $\alpha$-SMA, vimentin, and MMP-9 expression and decreasing E-cadherin expression, with changes in cell morphology. TPA treatment induced EMT in a PKC-dependent manner. Moreover, the inhibition of aurora kinase $A$ by siRNAs and inhibitors (reversine and VX-680) suppressed TPA-induced cell invasion, migration, and EMT in SW480 human colon cells. Inhibition of aurora kinase A blocked TPA-induced vimentin and MMP-9 expression, and decreased E-cadherin expression. Furthermore, the knockdown of aurora kinase $A$ decreased the transcriptional activity of NF-KB and AP-1 in PKC-stimulated SW480 cells. These findings indicate that aurora kinase $\mathrm{A}$ induces migration and invasion by inducing EMT in SW480 colon cancer cells. To the best of our knowledge, this is the first study that showed aurora kinase A is a key molecule in PKC-induced metastasis in colon
\end{abstract}

*Corresponding authors. Eun Yong Chung, Tel: +82-32-340-7076; Fax: +82-32-340-2255; E-mail: anes36@catholic.ac.kr; Jong-Suk Kim, Tel: +82-63-270-3085; Fax: +82-63-274-9833; E-mail: jsukim@jbnu.ac.kr ${ }^{\text {\#}}$ These authors contributed equally to this work.

https://doi.org/10.5483/BMBRep.2022.55.2.169

Received 24 November 2021, Revised 30 November 2021, Accepted 1 December 2021

Keywords: Aurora kinase A, Colon cancer, Epithelia-to-mesenchymal transition, Matrix metalloproteinase-9, Protein kinase C cancer cells. [BMB Reports 2022; 55(2): 87-91]

\section{INTRODUCTION}

Aurora kinase is a family of serine/threonine kinases associated with mitotic progression. The increase of aurora kinases is normally detected in cancers. Recent data indicate that aurora kinase A elevates distant metastasis by inducing EMT in breast cancer cells (1-5). Therefore, aurora kinases have become hopeful therapeutic targets for cancer treatment.

Colorectal cancer (CRC) is frequently diagnosed in men and the second in women, and metastasis is the leading cause of cancer-related deaths $(6,7)$. EMT is when epithelial cells transdifferentiate into mesenchymal cells during embryonic development $(8,9)$. As a consequence, epithelial cells adopt the morphology of mesenchymal stromal cells and become motile and invasive. In cancer, EMT induces the migration and invasiveness of cancer cells, increasing their metastatic potential. Following this transformation, a change in expressions of cell surface proteins (E-cadherin, vimentin, and $\alpha$-SMA) has been observed (10, 11).

PKC is known to be a major factor in signal transduction. PKC activation promotes carcinogen-induced tumorigenesis and is associated with specialized cellular functions, including adhesion, invasion, and metastasis (12). Treatment with 12-O-tetradecanoylphorbol-13-acetate (TPA) induced cell migration and invasion in cancer cells (13). Recently, it has been reported that PKC activation includes the regulation of EMT (14-16). Although studies have implicated PKC in EMT, the underlying molecular mechanisms remain poorly understood. We recently studied that aurora kinase positively regulates TPA-induced invasion and MMP-9 expression of breast cancer cells (15).

Here, we show the involvement of aurora kinase $A$ in PKC-induced EMT in CRC cells. Treatment with TPA altered the expression EMT markers, such as increased vimentin, $\alpha$-SMA, and MMP-9, and decreased E-cadherin expression, accompanying changes in cell morphology. However, the silencing of enzyme 
by siRNAs and aurora kinase A inhibitors inhibited TPA-induced cell invasion, migration, and EMT in SW480 human colon cells. These results indicate that aurora kinase $\mathrm{A}$ induces migration and invasion by inducing EMT in SW480 colon cancer cells.

\section{RESULTS}

\section{PKC treatmemt induced EMT in SW480 cells}

SW480 colon cells were derived from a primary tumor and had no metastatic properties. To determine whether TPA induces EMT in colon cancer cells, we treated SW480 cells with TPA. Treatment of cells with TPA induced changes in EMT markers, such as increased $\alpha$-SMA, vimentin, MMP-9 expression and decreased E-cadherin with changes in cell morphology (Fig. 1A, $\mathrm{B})$. We then confirmed the migration and invasion potential of cells treated with TPA, and observed that cells developed strong migration and invasive ability after $24 \mathrm{~h}$ of TPA treatment (Fig. 1C). Next, to investigate whether the induction of EMT by TPA is PKC-dependent, cells were treated with GF 109203X, a PKC inhibitor, $1 \mathrm{~h}$ before TPA treatment. Pretreatment with GF 109203X blocked TPA-induced EMT (Fig. 1C, D). These results confirmed that TPA induced EMT in a PKC-dependent manner.

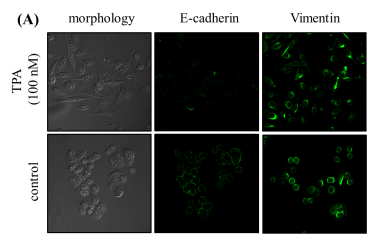

(B)

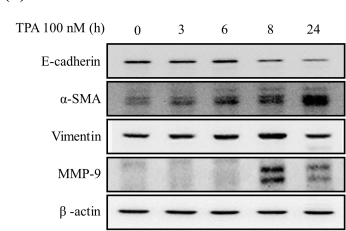

(C)

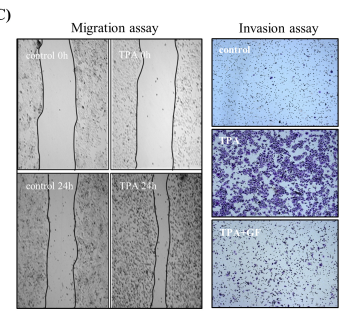

(D)

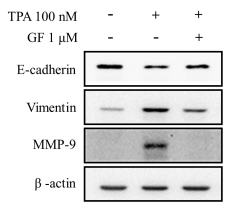

Fig. 1. $P K C$ is a potent inducer of EMT in colon cancer cells. (A) SW480 cells were treated with $100 \mathrm{nM}$ TPA for $24 \mathrm{~h}$ and then photographed to determine cell morphology. E-cadherin (green), vimentin (green), and nuclear DNA (blue; DAPI) were stained. (B) Cells were treated with $100 \mathrm{nM}$ TPA for the indicated times. To determine their protein expression, EMT markers (E-cadherin, $\alpha$-SMA, vimentin, and MMP-9) were analyzed via western blot. $\beta$-actin was used as a loading control. (C) GF pretreatment for $1 \mathrm{~h}$ and after treatment with TPA for $24 \mathrm{~h}$. For the cell invasion assay, invading cells that passed through the Matrigel coating chamber were stained with toluidine blue. SW480 cells were plated in a 6-well plate and a scratch migration assay was performed. Cells were scratched at $0 \mathrm{~h}$ and photographed at $0 \mathrm{~h}$ and $24 \mathrm{~h}$. (D) Cells were treated with GF for 1 $h$ and then with TPA. After $24 \mathrm{~h}$, cell lysates were analyzed via western blotting to determine the protein expression of E-cadherin, vimentin, MMP-9. $\beta$-actin was used as a loading control.

\section{Aurora kinase A is involved in PKC-induced EMT in SW480 cells}

We explored whether the expression of aurora kinase A was altered in SW480 cells after exposure to TPA. TPA treatment increased aurora kinase A expression in SW480 cells (Fig. 2A). Therefore, the role of aurora kinase A in PKC-induced EMT was examined in SW480 cells. The protein expression of aurora kinase $\mathrm{A}$ was determined to confirm that it was silenced by siRNA treatment (Fig. 2B). Next, we examined the alteration of EMT markers induced by aurora kinase A expression. The inhibition of aurora kinase A using siRNA and specific inhibitors (reversine and VX-680) suppressed the alteration in expressions of vimentin, MMP-9, and E-cadherin (Fig. 2C, D). Therefore, these results suggest that aurora kinase A plays a major role in PKCinduced EMT in colon cells.

\section{Aurora kinase A regulates PKC-induced cell invasion and migration in SW480 cells}

To investigate the role of aurora kinase A in PKC-induced cell invasion and migration of SW480 cells, we performed in vitro Matrigel invasion and scratch migration assays. The inhibition of aurora kinase A using siRNA suppressed TPA-induced invasion and migration (Fig. 3A). Additionally, we checked the effect of aurora kinase inhibitors such as reversine and VX680 in cell invasion. We observed that both agents inhibited TPA-stimulated cell invasion (Fig. 3B). These results suggest that the silencing of aurora kinase A suppressed PKC-induced invasion and migration in colon cells.
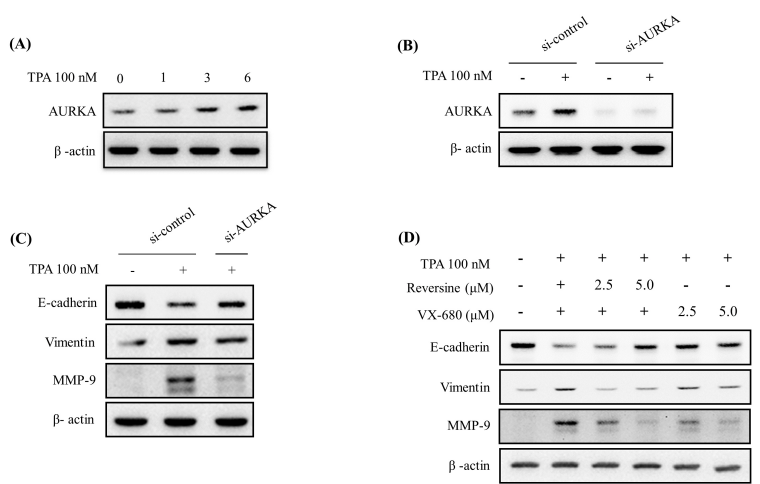

Fig. 2. Expression of AURKA during TPA-induced EMT in SW480 colon cancer cells. (A) Cells were treated with $100 \mathrm{nM}$ TPA for the indicated times. Expression of AURKA was analyzed via western blotting. (B) Expression of AURKA in SW480 cells transfected with AURKA siRNA was confirmed via western blotting. (C) Cells were transfected with AURKA-siRNA for $24 \mathrm{~h}$ and then treated with TPA for $24 \mathrm{~h}$. The expression of E-cadherin, vimentin, and MMP-9 were analyzed using western blotting. (D) SW480 cells were pretreated with AURK inhibitors (reversine and VX-680) for $1 \mathrm{~h}$ and then stimulated with TPA for $24 \mathrm{~h}$. The expressions of EMT markers were determined via western blotting. All western blots used $\beta$-actin as the loading control. 
(A)

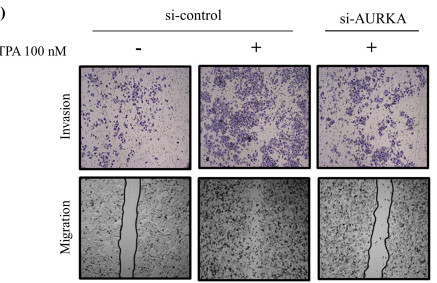

(B)

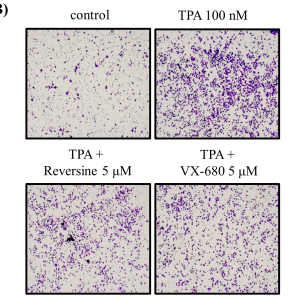

Fig. 3. AURKA regulates TPA-induced invasion and migration in SW480 colon cancer cells. (A) Cells were transfected with AURKAsiRNA and were transferred into a 24-well chamber and treated with $100 \mathrm{nM}$ TPA. After $24 \mathrm{~h}$, the cells were fixed and stained with a toluidine blue solution. For the scratch migration assay, confluent cell monolayers were scratched at $0 \mathrm{~h}$. (B) Cells were seeded in a 24-well chamber and treated with $100 \mathrm{nM}$ TPA. After $24 \mathrm{~h}$, the cells were fixed and stained with a toluidine blue solution.

\section{Aurora kinase A regulates PKC-induced NF- $\mathrm{KB} / \mathrm{AP}-1$ activation in SW480 cells}

$\mathrm{NF}-\mathrm{KB}$ and $\mathrm{AP}-1$ are major transcription factors that regulate EMT $(17,18)$. As shown in Fig. 4, TPA increased p65 (NF-KB subunit) expression and levels of phosphorylated c-Jun (AP-1 subunit) in the nucleus. To elucidate whether aurora kinase $A$ expression was involved in NF-KB/AP-1 activation, suppression of aurora kinase A in SW480 cells by treatment with siRNA or inhibitors was performed. Treatment was done after exposing the cells to TPA for $3 \mathrm{~h}$, and then the total nuclear protein was extracted to examine transcription factor activity. Inhibition of aurora kinase A suppressed TPA-induced expression of p65 and p-c-Jun in the nucleus (Fig. 4A, B). Indeed, TPA-induced NF- $\kappa B$ DNA-binding activity was partially blocked by treatment with aurora kinase inhibitors (Fig. 4C). These results indicate that aurora kinase A might control NF- $\mathrm{KB}$ and AP-1 in colon cells.

\section{DISCUSSION}

Aurora kinase plays an important role in maintaining chromosome stability as mitotic regulators. Moreover, recent data have shown that aurora kinase A activates EMT (19), and the stabilization and accumulation of aurora kinases, which drives the transition of breast cells from an epithelial to a highly invasive mesenchymal phenotype (20). Aurora kinase expression in patients with CRC liver metastases is associated with poor prognosis $(21,22)$. These findings strongly indicate that aurora kinases are negative regulators of the highly invasive mesenchymal phenotype in cancer cells. Our study suggests that aurora kinase A induces migration and invasion by inducing EMT in SW480 cells. This study shows that aurora kinase A is key to PKC-induced metastasis and is a novel therapeutic molecule in SW480 colon cancer cells.

EMT is a stage of phenotypic alteration in cancer cells, in which cells undergo morphological transformation. During EMT, cells lose their epithelial feature and get a mesenchymal phenotype. Phenotypic changes are thought to be derived from a shift

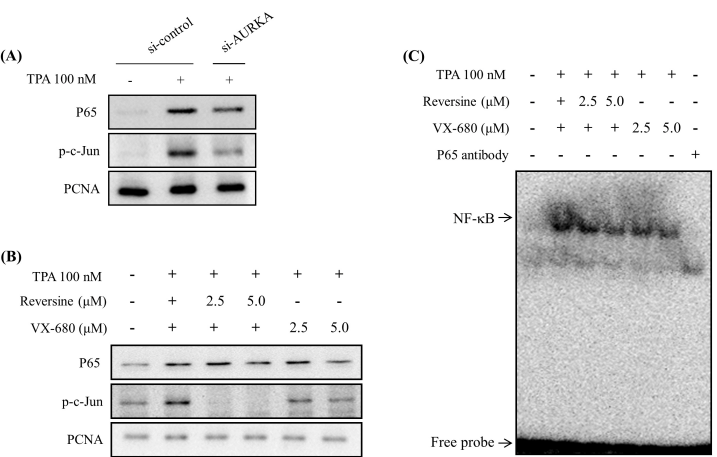

Fig. 4. AURKA inhibits TPA-induced NF- $\mathrm{K} B$ and $A P-1$ activation in SW480 colon cancer cells. (A) Cells were transfected with AURKAsiRNA for $24 \mathrm{~h}$ and then treated with TPA for $3 \mathrm{~h}$. The expressions of p65 and p-c-Jun were analyzed using western blotting. PCNA was used as the nuclear loading control. (B) SW480 cells were pretreated with AURK inhibitors (reversine and VX-680) for $1 \mathrm{~h}$ and then stimulated with TPA for $3 \mathrm{~h}$. Cell nuclear extracts were obtained and subjected to western blotting to determine the nuclear levels of NF- $\kappa B$ (p65) and AP-1 (p-c-Jun) subunits. PCNA was used as the nuclear loading control. (C) Cells were treated with AURK inhibitors (reversine and VX-680) for $1 \mathrm{~h}$ and then stimulated with TPA for $3 \mathrm{~h}$. The DNA binding activity of NF- $\mathrm{KB}$ was determined through an electrophoretic mobility gel shift assay (EMSA).

in the expression between epithelial (E-cadherin) and mesenchymal (Vimentin, Snail, and Twist-1) factors $(8,9)$. EMT markers have been a major potential target for the prevention and treatment of various human cancers $(19,22)$. As a result, the transition from an epithelial to a mesenchymal phenotype in cancer cells increases their capability to invade and migrate to around tissues.

MMP-9 plays a major role in metastasis $(23,24)$. In addition, the induction of invasiveness and EMT has been associated with the activation of NF-KB and AP-1 in cancer cells (25-27), suggesting that MMP-9 expression leads to EMT in cancer. However, the signaling systems that lead to EMT in colon cancer cells remain unclear. A previous study found that the aurora kinases regulates TPA-induced MMP-9 expression in breast cancer cells (15). This suggests that aurora kinases is also associated with PKC-induced MMP-9 expression in colon cancer cells.

In this study, we found that the silencing of aurora kinases A by their respective siRNAs and inhibitors blocked TPA-induced cell invasion, migration, MMP-9 expression, and EMT in SW480 cells (Figs. 2 and 3). These results show that aurora kinase stimulates migration and invasion by inducing EMT and MMP-9 expression in SW480 cells. Furthermore, the knockdown of aurora kinase $A$ reduced the transcriptional activity of NF- $\mathrm{\kappa B}$ and AP-1 in PKC-mediated SW480 cells (Fig. 4). Our results indicate that aurora kinase plays a role in PKC-induced MMP-9 expression through the NF- $\mathrm{KB}$ and AP-1 signaling pathways in SW480 cells. Supporting this hypothesis, another study indicated that aurora kinase is an important target in cancer through the inhibition of the EMT pathway (19). These results suggest that 
aurora kinase is a key regulator of EMT in SW480 colon cancer metastasis.

In conclusion, our study shows that MMP-9 expression and the metastasis of colon cancer cells may be modulated via an aurora kinase A-induced EMT signaling mechanism. Our data showed that aurora kinase A might be a target for the prevention and treatment of colon cancer.

\section{MATERIALS AND METHODS}

\section{Cell culture and materials}

SW480 cells were purchased from the Korean Cell Line Bank. The cells were cultured in high-glucose DMEM containing $10 \%$ bovine serum albumin and $1 \%$ antibiotics $(10,000 \mathrm{U} / \mathrm{ml}$ penicillin and $10,000 \mu \mathrm{g} / \mathrm{ml}$ streptomycin) at $37^{\circ} \mathrm{C}$ in an incubator with $5 \% \mathrm{CO}_{2}$. TPA, DMSO, and $\beta$-actin antibodies were obtained from Sigma-Aldrich (St. Louis, MO, USA). GF 109203X and reversine were obtained from Calbiochem (St. Louis, MO, USA). VX-680 was purchased from Selleck (Houston, TX, USA). Primary antibodies against $\alpha$-SMA, vimentin, MMP-9, E-cadherin, p65, and PCNA were purchased from Santa Cruz (CA, USA), and aurora kinase $A(A \cup R K A)$ and p-c-Jun were obtained from Cell Signaling Technology (Beverly, MA, USA).

\section{Western blotting}

SW480 cell extracts were isolated using M-PER ${ }^{\circledR}$ Mammalian Protein Extraction Reagent (Thermo Fisher Scientific, Waltham, MA, USA) supplemented with a protease inhibitor (Millipore, Billerica, MA, USA). Twenty micrograms of protein was separated using $7-12 \%$ SDS-PAGE and transferred onto PVDF membranes (GE Healthcare Life Sciences, Buckinghamshire, UK). Membranes were incubated with the corresponding primary antibodies at $4^{\circ} \mathrm{C}$ for overnight and HRP-conjugated secondary antibodies at $4{ }^{\circ} \mathrm{C}$ for $1 \mathrm{~h}$. HRP was detected using an imaging analyzer (LAS 1000; FujiFilm Corporation, Japan).

\section{Immunofluorescence analysis}

Cell fixation was carried out with $4 \%$ formalin for $30 \mathrm{~min}$ at room temperature, and cell permeability was measured using $0.2 \%$ Triton-X100 in PBS for 20 min. Next, the cells were blocked with $2 \%$ bovine serum albumin in PBS to prevent nonspecific antibody binding. The cells were then incubated with anti-Ecadherin and anti-vimentin antibodies, followed by Alexa Fluor-400 conjugated secondary antibodies (Molecular Probes, Eugene, OR, USA). Confocal images were obtained using a Carl Zeiss laser confocal microscope (Model no. LSM 510 META).

\section{Matrigel invasion assay}

Invasion assays were conducted using a Transwell insert chamber ( $8 \mu \mathrm{m}$ pore size) coated with Matrigel (BD Biosciences, Franklin Lakes, NJ, USA). SW480 cells were added to the upper chamber, and a chemoattractant was added to the bottom well. The wells were filled with a conditioned medium containing TPA for 24 h. After incubation, the invading cells were fixed, stained with toluidine blue, and photographed (40× magnification). Three independent experiments were conducted.

\section{Scratch migration assay}

A scratch migration assay was performed to compare the migratory abilities of normal and aurora kinase A (AURKA)-knockdown SW480 cells. All cells were cultured to confluency, scratched, photographed at $0 \mathrm{~h}$, and then photographed after treatment with TPA for $24 \mathrm{~h}$ using a light microscope at $40 \times$ magnification. All experiments were performed in triplicate.

\section{Electrophoretic motif shifting assay}

Nuclear proteins were extracted using NE-PER ${ }^{\circledR}$ Nuclear and Cytoplasmic Extraction Reagents (Pierce Biotechnology, Rockford, IL, USA). Extraction was performed according to the manufacturer's protocol. Oligonucleotides containing the $\kappa$-chain $(\kappa B$, 5'-CCGGTTAACAGAGGGGGCTITCCGAG-3') binding site were synthesized and used as probes for the gel retardation assays. The NF- $\kappa B$ oligonucleotide was labeled with [ $\alpha-32 \mathrm{P}] \mathrm{dCTP}$ using a Rediprime II DNA Labeling System (Amersham Life Science, Amersham, UK). In competition assays, a 100-fold excess of cold $\kappa \mathrm{B}$ oligonucleotide was added. The DNA-protein complexes were analyzed via electrophoresis on a $4 \%$ polyacrylamide gel. After electrophoresis, the gel was dried and examined using autoradiography.

\section{ACKNOWLEDGEMENTS}

This study was supported by the Biomedical Research Institute, Jeonbuk National University Hospital, and the Basic Science Research Program through the National Research Foundation of Korea (NRF), funded by the Ministry of Education (NRF-2019 R1A2C1003454 and 2020R1I1A1A01054100).

\section{CONFLICTS OF INTEREST}

The authors have no conflicting interests.

\section{REFERENCES}

1. Privette LM and Petty EM (2008) CHFR: a novel mitotic checkpoint protein and regulator of tumorigenesis. Transl Oncol 1, 57-64

2. Carvajal RD, Tse A and Schwartz GK (2006) Aurora kinases: new targets for cancer therapy. Clin Cancer Res 12, 68696875

3. Yu X, Minter-Dykhouse K, Malureanu L et al (2005) Chfr is required for tumor suppression and Aurora $A$ regulation. Nat Genet 37, 401-406

4. Tang A, Gao K, Chu L, Zhang R, Yang J and Zheng J (2017) Aurora kinases: novel therapy targets in cancers. Oncotarget 8, 23937-23954

5. Du R, Huang C, Liu K, Li X and Dong Z (2021) Targeting AURKA in cancer: molecular mechanisms and opportunities for cancer therapy. Mol Cancer 20, 15 
6. Jemal A, Center MM, DeSantis C and Ward EM (2010) Global patterns of cancer incidence and mortality rates and trends. Cancer Epidemiol Biomarkers Prev 19, 18931907

7. Siegel R, Naishadham D and Jemal A (2013) Cancer statistics, 2013. CA Cancer J Clin 63, 11-30

8. Mathias RA, Gopal SK and Simpson RJ (2013) Contribution of cells undergoing epithelial-mesenchymal transition to the tumour microenvironment. J Proteomics 78, 545-557

9. Thiery JP and Sleeman JP (2006) Complex networks orchestrate epithelial-mesenchymal transitions. Nat Rev Mol Cell Biol 7, 131-142

10. Batlle E, Sancho E, Franci $C$ et al (2000) The transcription factor snail is a repressor of E-cadherin gene expression in epithelial tumour cells. Nat Cell Biol 2, 84-89

11. Usman S, Waseem NH, Nguyen TKN et al (2021) Vimentin is at the heart of epithelial mesenchymal transition (emt) mediated metastasis. Cancers (Basel) 13, 4985

12. Parekh DB, Ziegler W and Parker PJ (2000) Multiple pathways control protein kinase $\mathrm{C}$ phosphorylation. $\mathrm{EMBO}$ J 19, 496-503

13. Tarafdar A and Michie AM (2014) Protein kinase C in cellular transformation: a valid target for therapy? Biochem Soc Trans 42, 1556-1562

14. Masur K, Lang K, Niggemann B, Zanker KS and Entschladen F (2001) High PKC alpha and low E-cadherin expression contribute to high migratory activity of colon carcinoma cells. Mol Biol Cell 12, 1973-1982

15. Noh EM, Lee YR, Hong OY, Jung SH, Youn HJ and Kim JS (2015) Aurora kinases are essential for PKC-induced invasion and matrix metalloproteinase-9 expression in MCF-7 breast cancer cells. Oncol Rep 34, 803-810

16. Serova M, Ghoul A, Benhadji KA et al (2006) Preclinical and clinical development of novel agents that target the protein kinase C family. Semin Oncol 33, 466-478

17. Julien S, Puig I, Caretti E et al (2007) Activation of NFkappaB by Akt upregulates Snail expression and induces epithelium mesenchyme transition. Oncogene 26, 7445-7456
18. Nam EH, Lee Y, Moon B, Lee JW and Kim S (2015) Twist1 and AP-1 cooperatively upregulate integrin alpha5 expression to induce invasion and the epithelial-mesenchymal transition. Carcinogenesis 36, 327-337

19. D'Assoro AB, Haddad T and Galanis E (2015) Aurora-A kinase as a promising therapeutic target in cancer. Front Oncol 5, 295

20. D'Assoro, AB, Liu T, Quatraro C et al (2014) The mitotic kinase Aurora-A promotes distant metastases by inducing epithelial-to-mesenchymal transition in ERalpha $(+)$ breast cancer cells. Oncogene 33, 599-610

21. Goos JA, Coupe VM, Diosdado B et al (2013) Aurora kinase A (AURKA) expression in colorectal cancer liver metastasis is associated with poor prognosis. Br J Cancer 109, 2445-2452

22. Steeghs N, Eskens FA, Gelderblom H et al (2009) Phase I pharmacokinetic and pharmacodynamic study of the aurora kinase inhibitor danusertib in patients with advanced or metastatic solid tumors. J Clin Oncol 27, 5094-5101

23. Bauvois B (2012) New facets of matrix metalloproteinases MMP-2 and MMP-9 as cell surface transducers: outside-in signaling and relationship to tumor progression. Biochim Biophys Acta 1825, 29-36

24. Stetler-Stevenson WG (1999) Matrix metalloproteinases in angiogenesis: a moving target for therapeutic intervention. J Clin Invest 103, 1237-1241

25. Nomura A, Majumder K, Giri B et al (2016) Inhibition of NF-kappa B pathway leads to deregulation of epithelialmesenchymal transition and neural invasion in pancreatic cancer. Lab Invest 96, 1268-1278

26. Julien S, Puig I, Caretti E et al (2007) Activation of NFkappaB by Akt upregulates Snail expression and induces epithelium mesenchyme transition. Oncogene 26, 74457456

27. Bakiri L, Macho-Maschler S, Custic I (2015) Fra-1/AP-1 induces EMT in mammary epithelial cells by modulating Zeb1/2 and TGF $\beta$ expression. Cell Death Differ 22, 336-350 